\title{
Forward acoustic scattering by moving objects: Theory and experiment
}

\author{
LEI Bo*, YANG KunDe, MA YuanLiang \& WANG Lu \\ Institute of Acoustic Engineering, Northwestern Polytechnical University, Xi'an 710072, China
}

Received August 1, 2011; accepted September 18, 2011

\begin{abstract}
The normal mode model for scattering in shallow water is employed to investigate the forward scattering with a target crossing the source-receiver axial line. An experiment was conducted in a littoral environment to analyze forward scattering by a slowly moving object. The theoretical and experimental results show that the sound field aberration takes minimum values if the object is located mid-point along the source-receiver line, whereas it attains its maximum if the object is close to the source or receiver. The total field is either enhanced or suppressed if the object crosses different Fresnel zones. In addition, the duration of shadow-induced aberration is dependent on the width of the first Fresnel zone, which is longest at the mid-point of the source-receiver line.
\end{abstract}

forward scattering, moving object, Fresnel zone, shadow effect, sound field aberration

Citation: $\quad$ Lei B, Yang K D, Ma Y L, et al. Forward acoustic scattering by moving objects: Theory and experiment. Chin Sci Bull, 2012, 57: 313-319, doi: 10.1007/s11434-011-4880-6

Bistatic sonar can be employed for target detection outside an ellipse surrounding the source and receiver. However, when the target is directly located between the source and receiver, it is particularly hard to obtain useful detection due to the intense direct blast of the incident field, the so-called "looking into the sunlight" problem [1,2]. Using traditional processing techniques, this direct blast can overwhelm any signature from a target crossing the source-receiver line, potentially masking the target.

In spite of an object's stealth ability, forward scattering is much stronger than backward scattering [3,4]. Forward scattering from an intrusive object could cause aberration in the waveguide response, providing an advantage in quietobject detection [5-8]. Transient aberration results from interference between the incident and scattered fields from an object [9], and its features can be actually difficult to be predicted in a multipath environment. Depending on the source-receiver geometry, the presence of a scatterer can potentially cause both enhancement (constructive interfer-

*Corresponding author (email: lei.bo@nwpu.edu.cn) ences with the scattered field) or suppression (shadow effect) of the amplitude of the received signals [10]. Perturbations of the received field caused by objects moving into this field can be used for detection if (1) the environmental variations in the received waveform remain relatively stable during the passage time of object and (2) the aberrations caused by the object intrusion are strong compared to the aforementioned environmental variability.

Over the past few years, a detection method using forward scattering techniques was proposed for moving underwater targets, and a successful lake experiment was conducted with a foam plastic target [5,6]. A detection and localization algorithm based on the same techniques was described and evaluated based upon the experiment in a harbor environment [7]. Song et al. [8] proposed an active time reversal approach, where variations caused by a crossing target were observed as rising levels in the side-lobes near the focal zone. Recently, a principal-component analysis of the stable portion of the recorded acoustic signals was used to determine crossing times of targets and to isolate some of its scattered wavefield components [10]. However, 
all these reported papers concentrated mainly on the detection of submerged objects, whereas the forward scattering process with moving objects was seldom discussed at long range compared to water depth.

Our investigation, reported in this paper, was to characterize the sound field aberrations caused by forward scattering from slowly-moving objects. The normal mode model $[11,12]$ is employed to simulate the forward scattering field with an object crossing the source-receiver line and a physical interpretation is given. Furthermore, we designed and conducted lake experiments at range-to-depth ratios up to about 190, much farther than reported experiments. The acoustic field aberrations caused by moving objects were clearly observed and agreed well with theoretical results.

\section{Theoretical analysis of acoustic forward scattering}

\subsection{Modeling forward scattering with normal mode model}

In a stratified waveguide, it is assumed that (1) the object is contained within a layer in which the sound speed is a constant; (2) multiple scattering between the waveguide boundaries and object can be neglected; and (3) the range $\rho_{0}$ from the object to the source and the range $\rho$ from the object to the receiver are large enough that the scattered field can be expressed as a linear function of the object's scattering function. Thus the scattered field at the receiver's position $\boldsymbol{r}(\rho, z)$ can be expressed as

$$
\begin{gathered}
\Phi_{s}(\boldsymbol{r})=\frac{(4 \pi)^{2}}{k} \sum_{m n}\left\{A _ { n } ( \boldsymbol { r } _ { 0 } ) \left[A_{m}(\boldsymbol{r}) S\left(\pi-\alpha_{m}, \beta ; \pi-\alpha_{n}, \beta_{i}\right)\right.\right. \\
\left.-B_{m}(\boldsymbol{r}) S\left(\alpha_{m}, \beta ; \pi-\alpha_{n}, \beta_{i}\right)\right] \\
-B_{n}\left(\boldsymbol{r}_{0}\right)\left[A_{m}(\boldsymbol{r}) S\left(\pi-\alpha_{m}, \beta ; \alpha_{n}, \beta_{i}\right)\right. \\
\left.\left.-B_{m}(\boldsymbol{r}) S\left(\alpha_{m}, \beta ; \alpha_{n}, \beta_{i}\right)\right]\right\}, \\
A_{m}(\boldsymbol{r})=i d\left(8 \pi k_{m} \rho\right)^{-1 / 2} u_{m}(z) N_{m} \mathrm{e}^{i\left(k_{m} \rho+\gamma_{m} H-\pi / 4\right)}, \\
B_{m}(\boldsymbol{r})=i d\left(8 \pi k_{m} \rho\right)^{-1 / 2} u_{m}(z) N_{m} \mathrm{e}^{i\left(k_{m} \rho-\gamma_{m} H-\pi / 4\right)}, \\
A_{n}\left(\boldsymbol{r}_{0}\right)=i d\left(8 \pi k_{n} \rho_{0}\right)^{-1 / 2} u_{n}\left(z_{0}\right) N_{n} \mathrm{e}^{i\left(k_{n} \rho_{0}+\gamma_{n} H-\pi / 4\right)}, \\
B_{n}\left(\boldsymbol{r}_{0}\right)=i d\left(8 \pi k_{n} \rho_{0}\right)^{-1 / 2} u_{n}\left(z_{0}\right) N_{n} \mathrm{e}^{i\left(k_{n} \rho_{0}-\gamma_{n} H-\pi / 4\right)},
\end{gathered}
$$

where $A_{m}(\boldsymbol{r})$ and $A_{n}\left(\boldsymbol{r}_{0}\right),\left(B_{m}(\boldsymbol{r})\right.$ and $\left.B_{n}\left(\boldsymbol{r}_{0}\right)\right)$ denote the down-going (up-going) plane wave amplitudes; $\boldsymbol{r}_{0}$ indicates the source's position; $k_{n}$ and $k_{m}$ are the $n$th and $m$ th horizontal wave number; $\gamma_{n}$ and $\gamma_{m}$ are the $n$th and $m$ th vertical wave number, respectively; $H$ is the depth of the object center from the sea surface, $d$ is the density, and $N$ is the mode amplitude. $S\left(\alpha, \beta ; \alpha_{i}, \beta_{i}\right)$ is the object's plane wave scattering function in free space, with $\left(\alpha_{i}, \beta_{i}\right)$ denoting the direction of incident normal mode, and $(\alpha, \beta)$ the direction of scattering wave propagation. The scattering function can be expressed by approximate analytical solutions for simple- shaped objects [13]. For forward scattering, we have $\alpha=\alpha_{i}$, $\beta=\beta_{i}$. According to the normal mode model, the mode amplitude function in the layer of object is

$$
u_{n}(z)=N_{n}\left(\mathrm{e}^{-i \gamma_{n}(z+H)}-\mathrm{e}^{i \gamma_{n}(z+H)}\right),
$$

and mode functions are normalized according to

$$
\delta_{n m}=\int_{-H}^{\infty} \frac{u_{m}(z) u_{n}^{*}(z)}{d(z)} \mathrm{d} z .
$$

In eq. (1), the field generated by the source is decomposed into modes incident on the object. Each incoming mode is composed of one down-going component with amplitude $A_{n}\left(\boldsymbol{r}_{0}\right)$ and incident elevation angle $\alpha_{n}$, and one up-going component with amplitude $B_{n}\left(\boldsymbol{r}_{0}\right)$ and incident elevation angle $\pi-\alpha_{n}$, respectively. Each scattered mode is also composed of a pair of plane waves, the down-going plane wave with amplitude $B_{m}(\boldsymbol{r})$ and elevation angle $\alpha_{m}$, the up-going plane wave with amplitude $A_{m}(\boldsymbol{r})$ and elevation angle $\pi-\alpha_{m}$ [12]. Each of the four terms in eq. (1) represents the coherent scattering of one of the two incoming plane wave components of the $n$th mode, into one of the two outgoing plane wave components of the $m$ th mode. The scattering process couples the modes so that the propagation and scattering are coherently convolved for objects submerged in a shallow water waveguide. The far-field interaction between the incident and scattered modes is determined by the scattering function which depends on the elevation angles of both the incident and scattered plane waves. The total scattered field is composed of the scattered fields from each incident plane wave.

We consider a Pekeris waveguide with $100 \mathrm{~m}$ water depth. With water density of $1 \mathrm{~g} / \mathrm{cm}^{3}$, the speed of sound is $1500 \mathrm{~m} / \mathrm{s}$. The lake floor is assumed to be a half-space acoustic medium with density $1.4 \mathrm{~g} / \mathrm{cm}^{3}$, speed of sound $1520 \mathrm{~m} / \mathrm{s}$, and a sound absorption coefficient of $0.15 \mathrm{~dB} / \lambda$. The source, at a depth of $50 \mathrm{~m}$, has frequency $1500 \mathrm{~Hz}$. The source-to-receiver distance is $8 \mathrm{~km}$. A plate $50 \mathrm{~m}$ in length and $6 \mathrm{~m}$ in width is at the same depth of $50 \mathrm{~m}$. The object crosses the mid-point perpendicularly between the source and receiver at different ranges. In eq. (1) the scattering function $S$ of this object is evaluated using Kirchhoff's diffraction integrals [14].

The results are shown in Figure 1, where the horizontal axis is the distance from the object to the source-receiver line. In Figure 1(a) and (c), the strengths of the forward scattering field at the depth of $10 \mathrm{~m}, 50 \mathrm{~m}$ and $90 \mathrm{~m}$ are shown. If the target approaches the source-receiver line (close to $0 \mathrm{~m}$ ), then clearly the forward scattering strength attains its maximum value. If the object leaves the sourcereceiver line, the scattering signals come from the sidelobes of the forward scattering. The total field, depicted in Figure 1(b) and (d), results from the interference of the forward scattering field and direct wave field. The forward scattering signal creates a destructive interference pattern 
with the direct wave as the target crosses the sourcereceiver line. As an exception, the total field at the depth of $10 \mathrm{~m}$ is enhanced in Figure 1(d) due to the phase reversal of the scattering wave within the multipath propagation. If the object is about $50 \mathrm{~m}$ far from the source-receiver line, the forward scattering signal interferes constructively with the direct wave, and the total field is enhanced slightly. If the object is far from the source-receiver line, the total field is close to the direct wave field because the scattering signal of side-lobe is much weaker than the direct wave. The theory underlying this forward scattering phenomenon is illustrated in Figure 2. If the target crosses the baseline, the path length between the direct wave and forward scattering signal is identical, but the signal's forward scattering phase is opposite to that of the direct signal, and the total signal has a minimum at this point. As the target continues to move, path differences increase, the signal's forward scattering phase is the same as that of the direct signal, and the total signal reaches its maximum. As the target progresses, the phase difference between forward scattering and direct signals is further increased and reaches $2 \pi$ at another point, the total signal becomes minimal again and the cycle repeats.

The results in Figure 1(b) show that if an object crosses the mid-point between source and receiver, the acoustic field aberration is about $1 \mathrm{~dB}$ at a depth of $50 \mathrm{~m}$ and the width of shadow-induced aberration is $100 \mathrm{~m}$. In contrast, if the object is close to the receiver as shown in Figure 1(d), the aberration is stronger than the results in Figure 1(b) with the width reduced to about $40 \mathrm{~m}$.

In practice, the shape of a typical target in shallow water can be simplified as spheroid. The environment still modeled as a Pekeris waveguide mentioned above. A rigid spheroid $60 \mathrm{~m}$ long and $8 \mathrm{~m}$ short axis is used instead of the plate. The spheroid is also at the depth of $50 \mathrm{~m}$. The object crosses perpendicularly the axial line between source and receiver at different ranges. The scattering function $S$ is evaluated using deformed cylinder method [15]. The simulated results shown in Figure 3 indicate that the forward scattering phenomenon of the spheroid is similar to that of the moving plate.

\subsection{Analyzing forward scattering with sonar equation}

Due to the complex acoustic coupling between the scattered beam pattern and the multipath propagation, it is complicated to predict directly using eq. (1) the forward scattering field of the object in a real environment at the receiver's location. However, the sonar equation can estimate the acoustic field aberration by forward scattering in the experimental environment [16]. Assuming cylindrical spreading in the waveguide, the transmission loss $T L$ is $10 \log _{10}(R)$ over the whole path distance $R$ between the source and
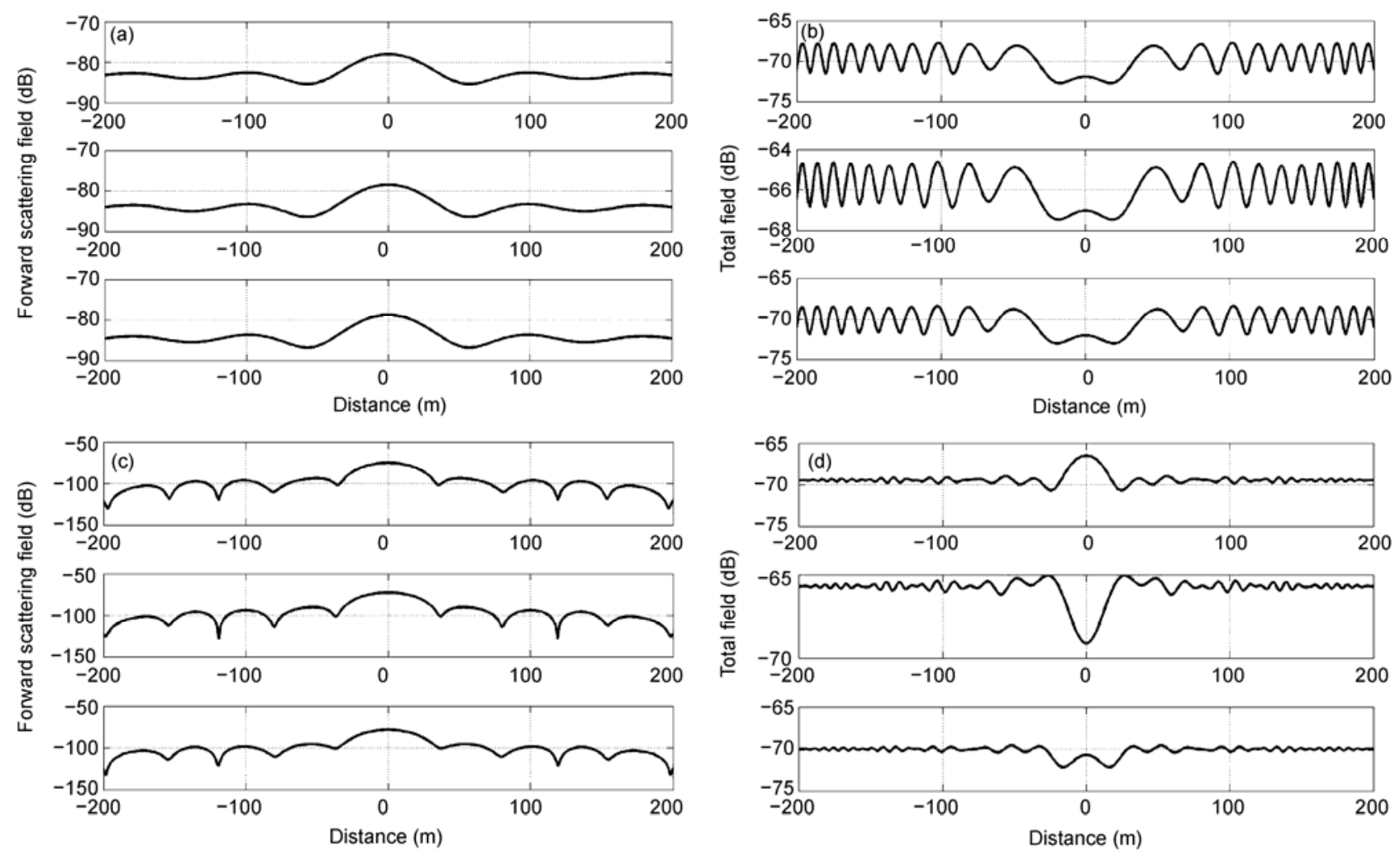

Figure 1 Forward scattering by a rigid plate in a Pekeris waveguide. From top to bottom, the panels in (a) and (c) are the forward scattering fields at $10 \mathrm{~m}$, $50 \mathrm{~m}$ and $90 \mathrm{~m}$. (b) and (d) are the total field strength corresponding to panels (a) and (c), respectively. The range from crossing-point of the target to receiver is $4 \mathrm{~km}$ for (a) and (b) and $1.5 \mathrm{~km}$ for (c) and (d). 


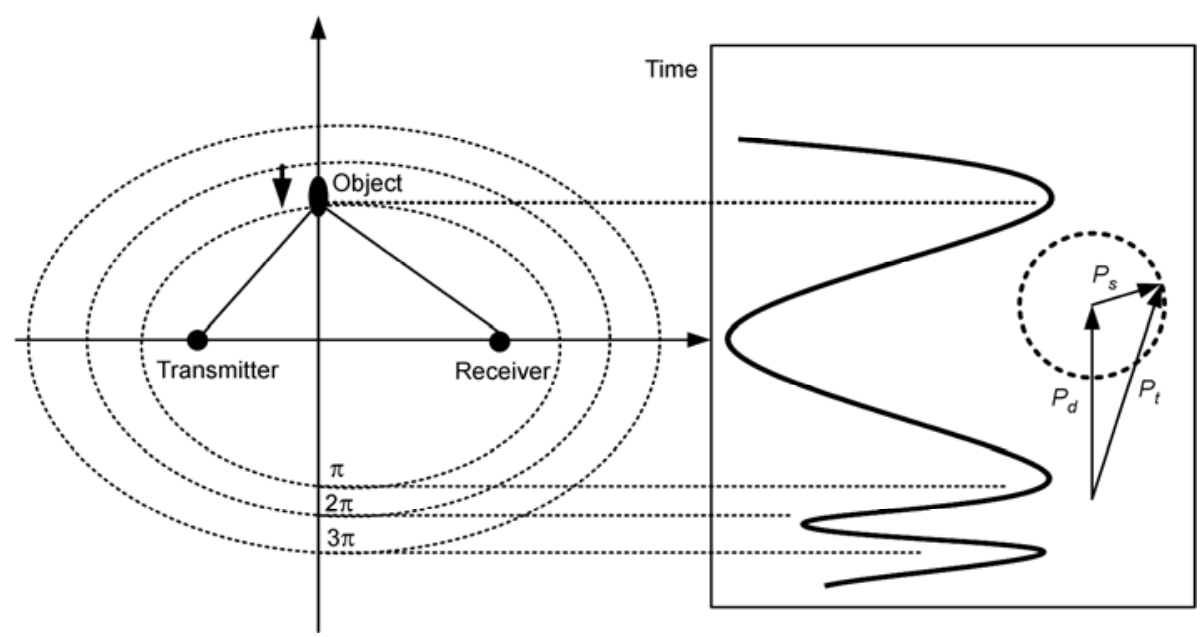

Figure 2 Illustration for forward scattering.

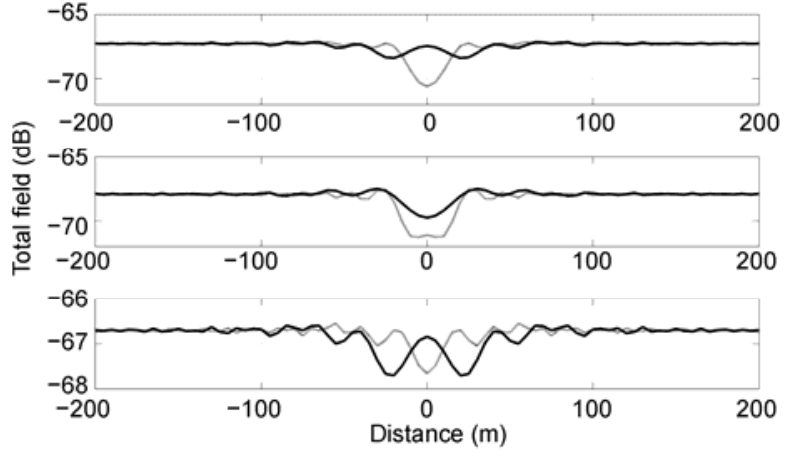

Figure 3 Forward scattering by a rigid spheroid in the Pekeris waveguide. The panels give, from top to bottom, the fields strengths at $10 \mathrm{~m}, 50 \mathrm{~m}$ and $90 \mathrm{~m}$. The solid line and dotted line correspond to ranges $4 \mathrm{~km}$ and $1.5 \mathrm{~km}$ to receiver, respectively.

receiver. In the absence of an object, the signal-to-noise ratio at the receiver can be estimated as

$$
S N R_{0}=S L-10 \log _{10}(R)+A G-N L,
$$

where $S L$ is the source level, $N L$ is the noise level, and $A G$ is the processing gain. Whereas, for an object with target strength $T S$ crossing at distance $D$ away from the receiver, we have

$$
S N R=S L-10 \log _{10}(R-D)+T S-10 \log _{10}(D)+A G-N L .
$$

In the far field, the forward target strength $T S$ associated with the projected cross-section area $A$ and wavelength $\lambda$ can be approximated by $20 \log _{10}(\mathrm{~A} / \lambda)$ [16], which is much stronger than the backward target strength [3]. Assuming that $N L$ remains constant during the target's crossing, the acoustic field aberration $\Delta S\left(S N R-S N R_{0}\right)$ at the receiver array can be approximated by

$$
\Delta S=20 \log _{10}(A / \lambda)+10 \log _{10}\left(\frac{R}{D \cdot(R-D)}\right) .
$$

If the object crosses the source-receiver line at crossing distance $D$, the radius of the first Fresnel zone for forward scattering of the far field can be expressed as

$$
R_{\mathrm{F}}=\sqrt{\lambda D(R-D) / R} .
$$

The forward scattering signal interferes with the direct blast destructively, when the distance between the target and source-receiver line is less than $R_{\mathrm{F}}$. If the object crosses the source-receiver line, the moving range of the object corresponding to destructive interference is $2 R_{\mathrm{F}}$, thus the duration of the SNR variation is determined by the speed of the object.

\section{Experimental investigation of forward scattering}

\subsection{Experiment setup}

The experiment, schematically depicted in Figure 4, was conducted in a lake characterized by a soft, muddy floor. The water depth was about $6 \mathrm{~m}$. An omnidirectional transmitter with resonance frequency $17 \mathrm{kHz}$ was deployed at a depth of $4 \mathrm{~m}$. A vertical line array, with 11 hydrophones equally spaced at $25 \mathrm{~cm}$, was moored along the water column. The bottom hydrophone was $2.4 \mathrm{~m}$ over the lake floor. A foam plastic plate $2 \mathrm{~m}$ long, $1 \mathrm{~m}$ wide and $6 \mathrm{~cm}$ thick, covered on both sides by aluminum plates (see right panel of Figure 4) was used target object. The center of the object was at a depth of about $2 \mathrm{~m}$. To provide negative buoyancy, a load was attached underneath. The object was towed $10 \mathrm{~m}$ behind the boat traveling approximately tangentially to the source-receiver line.

Because the object was small and the water was shallow, a higher frequency signal was transmitted for the experiment. A periodic 5-15 kHz LFM signal with $0.5 \mathrm{~s}$ sweep time was transmitted in succession. The speed of the tow boat was about $0.45 \mathrm{~m} / \mathrm{s}$. Based on visual observations, the 

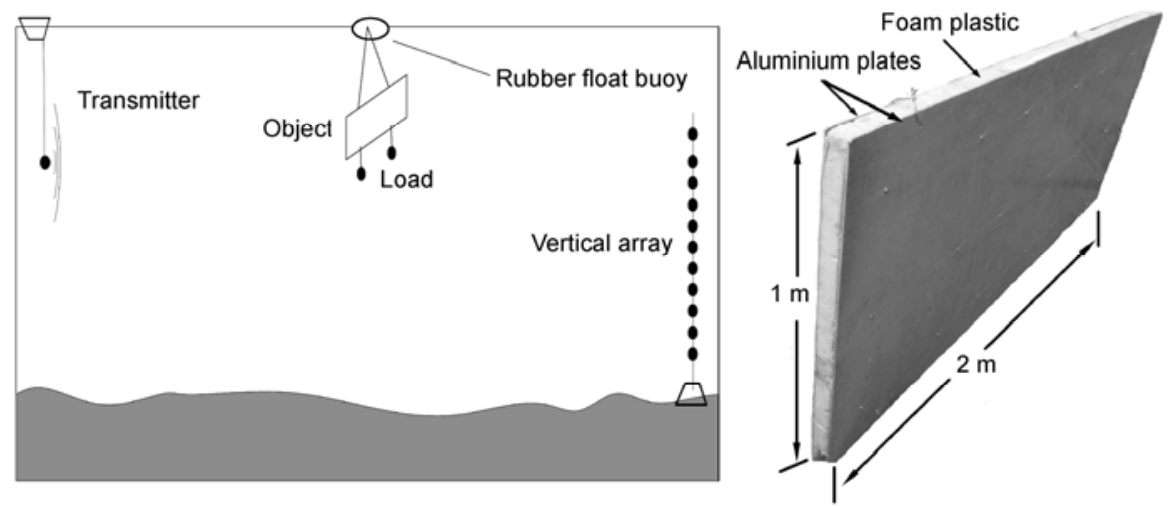

Figure 4 Experimental setup in the lake.

object crossed the source-receiver line along various nearly straight paths; these paths are shown in Figure 5. The time of crossing of the source-receiver line was determined to within $1 \mathrm{~s}$ accuracy, based on the GPS position of the rubber float buoy. The distance between the source and receiver array was $1145 \mathrm{~m}$, representing about 190 water depths.

According to eq. (7), if the object is crossing the mid-point between the source and receiver, the acoustic field aberration exhibits a minimum. In contrast, if the object is either close to the transmitter or the receiver, the field displays maximum aberration. For the object used in the experiment $\left(A=2 \mathrm{~m}^{2}, \lambda=0.15 \mathrm{~m}, T S \approx 22.5 \mathrm{~dB}\right)$, eq. (7) yields a theoretical estimate of $\Delta S=3.8 \mathrm{~dB}$ along the first path $(D=80 \mathrm{~m})$. Similarly, the estimated values are $-2 \mathrm{~dB}$ and 2.2 $\mathrm{dB}$ along the second $(D=534 \mathrm{~m})$ and third $(D=1025 \mathrm{~m})$ paths, respectively (see Table 1 ).

As mentioned by Sabra et al. [10], the sonar equation and free-space forward target strength values at a given frequency can only be used to provide a rough estimation of the acoustic aberration. The target in the waveguide is insonified by a fan of incident rays yielding range and depth variations such that target strength is different from the value in free space. Furthermore, the transmitted signal has broadband characteristics; thus the narrowband assumption of eqs. (5)-(7) is not satisfied fully.

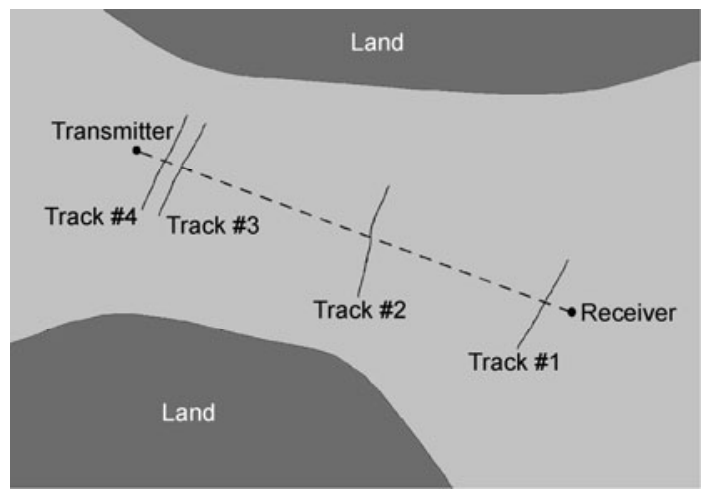

Figure 5 Top view schematic of the various paths for the forward scattering experiment between the source and receivers.
The acoustic aberration $\Delta S$ at different crossing distance $D$ is shown in Table 1, where the speed of the object is assumed to be $0.45 \mathrm{~m} / \mathrm{s}$. If the object is either close to the transmitter or receiver, the duration of aberration caused by forward scattering is short. In contrast, the longest duration is reached when the object is crossing at the mid-point between the transmitter and the receiver.

\subsection{Experimental results}

The analysis scheme for forward-scattering detection is shown in Figure 6. The first band-pass filter isolates perturbations from other environment sources in the direct field due to the passage of the object. The waveguide response is acquired by correlating the received signals with the transmitted signal and the strengths of stable arrivals are obtained as well. The median filter was found to be necessary due to strong interference from the direct blast. In Figure 6, the strengths of the stable arrival is calculated as the input signal $x(t)$ pass through the median filter, the aberration of the stable arrival perturbed by the crossing object is suppressed, and the strength of direct blast is obtained. Thus the acoustic field aberration can be acquired by extracting the strength of direct blast from the strength of the stable arrival. The normalized aberration in $\mathrm{dB}$ is expressed as

$$
y(t)=20 \log _{10}(x(t) / \tilde{x}(t)) .
$$

Executing this procedure on the recorded data can produces the normalized aberration for selected stable arrivals as typified by the arrival shown in Figure 7(a). With the

Table 1 The strength and duration of acoustic field aberration vs. crossing distance $D$

\begin{tabular}{rccc}
\hline$D(\mathrm{~m})$ & $\Delta S(\mathrm{~dB})$ & $R_{\mathrm{F}}(\mathrm{m})$ & Duration $(\mathrm{s})$ \\
\hline 80 & 3.8 & 3.3 & 14.7 \\
534 & -2.0 & 6.5 & 28.9 \\
1025 & 2.2 & 4.0 & 17.8 \\
1069 & 4.0 & 3.3 & 14.7 \\
\hline
\end{tabular}




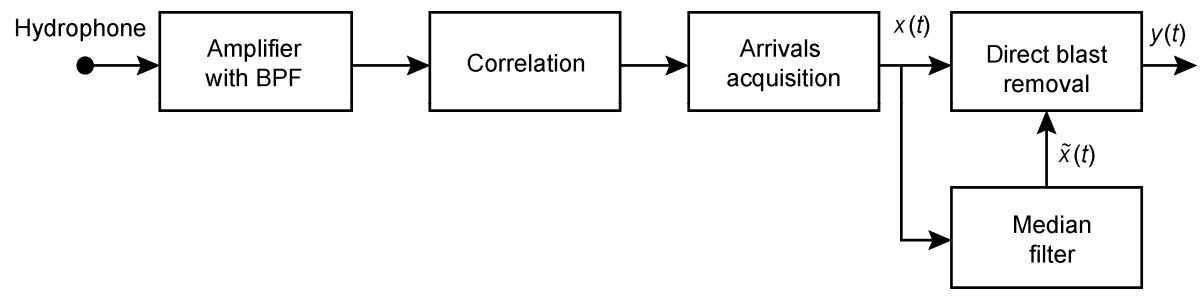

Figure 6 A scheme for forward scattering detection with wideband signal transmitted.
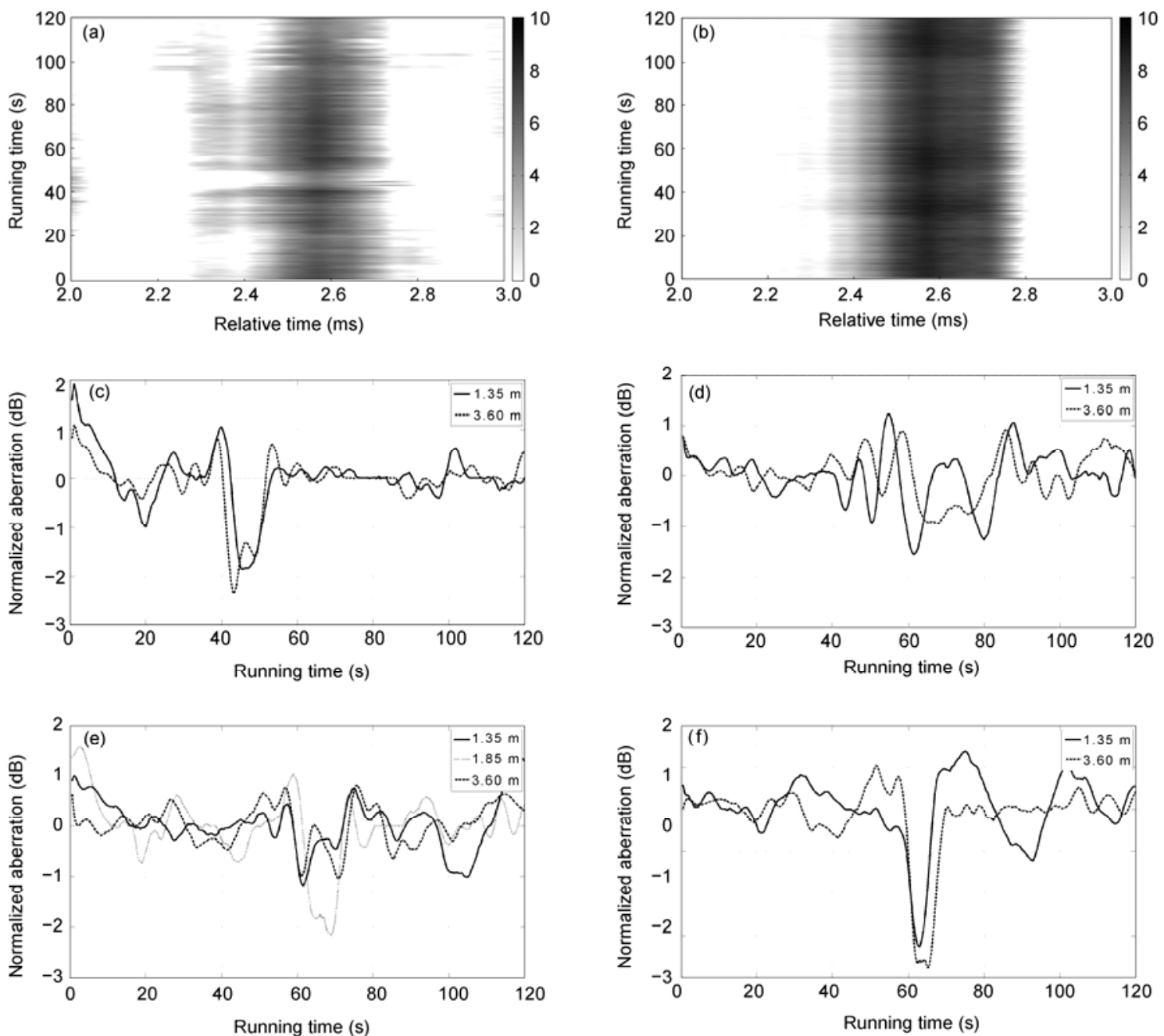

Figure 7 The acoustic aberration caused by forward scattering in different cases. (a) Multipath arrivals at a depth of $1.35 \mathrm{~m}$ with the object following the first path. (b) Multipath arrivals at a depth of $1.35 \mathrm{~m}$ without trailing object. (c)-(f) Aberrations with the object following Tracks \#1-\#4, respectively. The solid, dotted and dashed lines correspond to receiver depths of $1.35 \mathrm{~m}, 1.85 \mathrm{~m}$ and $3.60 \mathrm{~m}$, respectively.

object crossing the source-receiver line along the first path (see Figure 7(c)), the normalized aberrations at depths of $1.35 \mathrm{~m}$ and $3.6 \mathrm{~m}$ are $1.8 \mathrm{~dB}$ and $2.2 \mathrm{~dB}$ respectively, which is weaker than the theoretical value of $3.8 \mathrm{~dB}$. Along the second trajectory, the normalized aberration is about $1.6 \mathrm{~dB}$ at the depth of $1.35 \mathrm{~m}$ (see Figure 7(d)). For the third trajectory, the normalized aberration is about $2 \mathrm{~dB}$ at the depth of $1.85 \mathrm{~m}$, agreeing with theoretical estimates (see Figure 7(e)). With the object following the fourth path, the variation can attain $3.8 \mathrm{~dB}$ at the depth of $1.85 \mathrm{~m}$ (see Figure 7(f)). We remark that the normalized aberrations exhibit large values at the beginning of each run because the median filter does not reach steady state. From the normalized aberration along the four paths, it can be concluded that if the object is close to either the transmitter or receivers, aberrations become strong. Thus forward scattering detection provides an advantage in this situation.

Furthermore, the normalized aberrations are different at several depths due to multipath propagation. For instance, if an object crosses the source-receiver line along the third path, the aberrations are about $1 \mathrm{~dB}, 2 \mathrm{~dB}$ and $1 \mathrm{~dB}$ at depths of $1.35 \mathrm{~m}, 1.85 \mathrm{~m}$ and $3.6 \mathrm{~m}$, respectively (see Fig- 
ure 7(e)). The forward scattering includes the main-lobe and side-lobes. As the object is crossing the source-receiver line, destructive interference occurs between the main-lobe with the direct blast, generating the acoustic shadow. As the object leaves the source-receiver line, constructive interference is observed because the phase of scattered field is the same as that of direct field (see Figure 7(c)-(e)). This process is similar to that that underlies results shown in Figure 1. As the object leaves the source-receiver line, the received signal, displayed in Figure 7(c), is enhanced at about $38 \mathrm{~s}$ and $53 \mathrm{~s}$ into the run time, and suppressed between these times.

The durations of the shadow-induced aberration are about $14 \mathrm{~s}, 29 \mathrm{~s}, 16 \mathrm{~s}$ and $12 \mathrm{~s}$ at object crossings for the four trajectories, respectively (see Figure 7(c)-(f)). Clearly, experimental and theoretical results agree well. If the object is close to either source or receiver, the duration of the acoustic field aberration is shorter. In contrast, if the object crosses mid-point between source and receiver, the duration is maximal.

The effect of the moving boat on the received acoustic field is considered as well. Figure 7(b) displays the multipath arrivals of just the boat crossing the source-receiver line without the trailing object. The received acoustic field is seen to be stable and the moving boat has only a slight influence on that field. In other words, the aberrations of the received acoustic field observed in the experiment are mainly caused by the submerged object.

\section{Conclusions}

In this paper, we have given the normal mode model for a waveguide to analyze forward scattering from a target crossing the source-receiver line, and its physical significance. An experiment that we had conducted demonstrated the capability of forward scattering detection for slowmoving objects. The following conclusions are obtained:

(1) If an object crosses at mid-point between the source and receiver, the acoustic field aberration exhibits a minimum and the duration of shadow-induced aberration is longest. In contrast, if the object is close to either the transmitter or the receiver, maximal values for aberration will be observed due to direct-shadowing effects, although over a shorter duration.

(2) Forward scattering field was observed to yield either an enhancement or suppression of the total wavefield depending on the source-object-receiver geometry. If the object is within the first Fresnel zone, the total field is suppressed, otherwise it is enhanced.

(3) This study confirmed that our scheme for detection based on forward scattering is feasible in a reverberation littoral environment at ranges out to about 190 water depths. Additionally, it seems from the experiments that aberrations of the acoustic field caused by a moving target are more significant for deeper receivers near the lake floor.

These results show that there is significant potential for target detection by exploiting forward scattering phenomena. The lake experiment provided encouraging results. However, the variability of the received waveform must be fully understood and further improvements in the processing algorithm are necessary.

This work was supported by the National Natural Science Foundation of China (61101192, 11174235), the China Postdoctoral Science Foundation (20110491687), the Program for New Century Excellent Talents in University of China (NCET-08-0455), the Science and Technology Development Project of Shaanxi Province (2010KJXX-02) and the Foundation of State Key Laboratory on Acoustics (SKLOA201101).

1 Naluai N K, Lauchle G C. Bi-static sonar applications of intensity processing. J Acoust Soc Am, 2007, 121: 1909-1915

2 Gillespie B, Rolt K, Edelson G, et al. Littoral target forward scattering. Acoust Imaging, 1997, 23: 501-506

3 Lei B, Yang K D, Ma Y L. Physical model of the acoustic forward scattering by cylindrical shell with laboratory measurement. Chin Phys B, 2010, 19: 054301

4 Levy M F, Zaporozhets A A. Target scattering calculations with the parabolic equation method. J Acoust Soc Am, 1998, 103: 735-741

5 Matveev A L, Mityugov V V. Complex matched filtered of diffraction sound signals received by vertical array. Acoust Phys, 2000, 46: 80-86

6 Matveev L, Spindel R C, Rouseff D. Forward scattering observation with patially coherent spatial processing of vertical array signals in shallow water. IEEE J Ocean Eng, 2007, 32: 626-639

7 Folegot T, Martinelli G, Guerrini P. An active acoustic tripwire for simultaneous detection and localization of multiple underwater intruders. J Acoust Soc Am, 2008, 124: 2852-2860

8 Song H, Kuperman W A, Hodgkiss W S, et al. Demonstration of a high-frequency acoustic barrier with a time-reversal mirror. IEEE J Ocean Eng, 2003, 28: 246-249

9 Makris N C. A spectra approach to 3-D object scattering in layered media applied to scattering from submerged spheres. J Acoust Soc Am, 1998, 104: 2105-2113

10 Sabra K G, Conti S, Roux P, et al. Experimental demonstration of a high-frequency forward scattering acoustic barrier in a dynamic coastal environment. J Acoust Soc Am, 2010, 127: 3430-3439

11 Ingenito F. Scattering from an object in a stratified medium. J Acoust Soc Am, 1987, 82: 2051-2059

12 Ratilal P, Lai Y N, Makris N C. Validity of the sonar equation and Babinet's principle for scattering in a stratified medium. J Acoust Soc Am, 2002, 112: 1797-1816

13 Bowman J J, Senior T B A, Uslenghi P L E. Electromagnetic and Acoustic Scattering by Simple Shapes. Washington: Hemisphere Publishing Corporation, 1987

14 Hongo K, Sugaya H. Diffraction of an acoustic plane wave by a rectangular plate. J Appl Phys, 1997, 82: 2719-2729

15 Ye Z, Hoskinson E, Dewey R K, et al. A method for acoustic scattering by slender bodies. I. Theory and verification. J Acoust Soc Am, 1997, 102: 1964-1976

16 Urick R J. Principles of Underwater Sound. New York: McGraw-Hill, 1967. 306-327

Open Access This article is distributed under the terms of the Creative Commons Attribution License which permits any use, distribution, and reproduction in any medium, provided the original author(s) and source are credited. 